\title{
FATOR DE EXPANSÃO DE BIOMASSA E RAZÃO DE RAÍZES - PARTE AÉREA PARA Pinus spp. PLANTADAS NO SUL DO BRASIL
}

\author{
Ana Paula Dalla Corte ${ }^{1}$, Fernando da Silva ${ }^{2}$, Carlos Roberto Sanquetta ${ }^{3}$ \\ ${ }^{1}$ Eng $^{\mathrm{a}}$ Florestal, Dr ${ }^{\mathrm{a}}$., Depto. de Ciências Florestais, UFPR, Curitiba, PR, Brasil - anapaulacorte@ gmail.com \\ ${ }^{2}$ Eng. Florestal, Mestrando do Instituto Nacional de Pesquisas da Amazônia, Manaus, AM, Brasil - silvaflorestal@gmail.com \\ ${ }^{3}$ Eng. Florestal, Dr., Depto. de Ciências Florestais, UFPR, Curitiba, PR, Brasil - sanquetta@ufpr.br \\ Recebido para publicação: 24/06/2010 - Aceito para publicação: 07/08/2012
}

\begin{abstract}
Resumo
O fator de expansão da biomassa (FEB) e a razão de raízes (R) são usados na quantificação de carbono em florestas. Geralmente essas grandezas são consideradas constantes. 70 árvores foram usadas para demonstrar a correlação entre FEB e R e as variáveis dendrométricas e a idade. Doze modelos foram testados para FEB e R, e DAP, altura $(\mathrm{H})$ e idade. Foram estimados biomassa seca total, carbono e equivalente em $\mathrm{CO}_{2}$, com FEB e $\mathrm{R}$ default, valores médios e valores via equações. Obtiveram-se valores de $\mathrm{FEB}=1,47$ e de $\mathrm{R}=0,17$, o que significa que $47 \%$ da biomassa aérea correspondem à copa e $17 \%$ da biomassa total são raízes. A correlação entre FEB e $\mathrm{R}$ com as variáveis DAP, $\mathrm{H}$ e idade apresentaram-se significativos. As equações geradas foram eleitas como de melhor desempenho, tanto para FEB como para R. A comparação mostrou que o uso de valores fixos de FEB e R, seja o default do IPCC ou valores médios calculados com dados de campo, pode conduzir a erros. Concluiu-se que devem ser considerados FEB e $\mathrm{R}$ em função de variáveis dendrométricas DAP e $\mathrm{H}$ e idade, para se ter estimativas mais fidedignas da biomassa, do carbono fixado e do carbono equivalente em $\mathrm{CO}_{2}$.

Palavras-chave: Alometria; carbono; regressão; MDL; modelagem.
\end{abstract}

\begin{abstract}
Biomass expansion factor and root-to-shoot ratio for Pinus spp. in southern Brazil. Biomass expansion factor (BEF) and root-to-shoot ratio (R) are variables for quantification of carbon in forests. These are considered as constant values in most studies. 70 sample trees were used to BEF and $\mathrm{R}$, and $\mathrm{DBH}$, tree height and age. Dry total biomass, carbon and $\mathrm{CO}_{2}$ equivalent were simulated by using the IPCC default values of FEB and R, corresponding average calculated from data using in this study and as well as the values estimated by regression equations. The values of $\mathrm{FEB}=1.47$ and $\mathrm{R}=0.17$ in this study means that $47 \%$ of total biomass corresponds to tree crown and $17 \%$ of it comprises tree roots or below-ground biomass. The correlation BEF and R with tree DAP, H and age were significant. Some equations to FEB and $\mathrm{R}$ as functions of the tree variables revealed better performance than others, as they fitted better. The simulations indicated that use of fixed values of BEF and R, either IPCC default or average actual data may lead to unreliable of carbon and CDM projects. As result, FEB and $\mathrm{R}$ use in regression equations relating them to $\mathrm{DBH}$, tree height and age are fundamental to get reliable estimates of tree biomass, carbon sink and $\mathrm{CO}_{2}$ equivalent.

Keywords: Allometry; carbon; regression; CDM; modeling.
\end{abstract}

\section{INTRODUÇÃO}

O aquecimento global decorrente da emissão de gases de efeito estufa (GEE) e seus impactos são temas importantes para a comunidade científica. Visando limitar as emissões de GEE, o Protocolo de Quioto foi assinado e entrou em vigor para que as partes signatárias pudessem implementar medidas válidas de redução ou captura de GEE da atmosfera, através de mecanismos de flexibilização. Entre esses mecanismos encontra-se o mecanismo de desenvolvimento limpo (MDL), por meio do qual países que ainda não possuem metas de redução de emissões podem ajudar os países com metas a alcançar os níveis de redução conforme preconiza o Protocolo de Quioto.

O MDL propicia que países em desenvolvimento, como o Brasil, que tem grande potencial de 
uso da terra, devido às extensas áreas desflorestadas e de terras com pastagem, agricultura e mineração, possam desenvolver projetos para diminuir suas emissões de GEE. Especificamente, o MDL engloba a modalidade florestamento/reflorestamento, que visa completar as questões relacionadas à mudança no uso da terra e florestas. Para a modalidade florestal, até pouco tempo atrás não existiam muitas metodologias e projetos submetidos ao conselho executivo do MDL, por diversos fatores, entre eles a dificuldade em realizar as estimativas e compreender todas as complexas questões biológicas envolvidas nesses ecossistemas. Constata-se, dessa forma, que a elaboração de um projeto de MDL florestal é um procedimento que requer muitos cálculos precisos acerca das remoções líquidas de GEE pelos sumidouros, bem como estimativas de reduções certificadas de emissões de GEE (CERs), o que não é uma tarefa simples.

Nos últimos anos, tivemos uma grande quantidade de metodologias aprovadas e projetos submetidos ao UNFCCC para subsidiar os projetos de MDL, entretanto, em geral, essas metodologias são confusas, difíceis de serem aplicadas e utilizam fatores genéricos (default). Algumas iniciativas de simplificação dessa metodologia, para que a comunidade em geral possa se valer das mesmas, foram realizadas, como é o caso do desenvolvimento da ferramenta denominada Ferramenta para Metodologias Aprovadas de Florestamento e Reflorestamento (TARAM, Tool for Afforestation and Reforestation Approved Methodologies). Outra iniciativa interessante foi proposta por Fernandes (2010), em que foi realizada a customização da ferramenta TARAM para projetos de MDL florestal com Pinus taeda no extremo sul do Paraná.

Para a realização das estimativas de biomassa e carbono nas florestas, existem diferentes abordagens. Geralmente, as metodologias separam-se em métodos diretos e métodos indiretos (SANQUETTA et al., 2004). Utilizar o método direto implica realizar medidas destrutivas, ou seja, realizar a coleta de biomassa em campo. O método indireto, mais empregado, baseia-se nas relações alométricas para apoiar as estimativas realizadas. Assim, variáveis de fácil mensuração (diâmetro a $1,30 \mathrm{~m}$, altura total, área basal) apoiam as estimativas de variáveis de difícil mensuração (volume, biomassa, carbono). Através da utilização desses métodos, é possível construir as estimativas de crescimento florestal em biomassa e carbono, para subsidiar a elaboração de projetos de MDL.

Quando são realizadas as estimativas de biomassa através do volume e densidade da madeira, em geral, necessita-se recorrer ao uso de Fatores de Expansão de Biomassa (FEB) para gerar as estimativas de biomassa aérea total (JOHNSON; SHARPE, 1983; KARJALAINEN; KELLOMÄKI, 1996; WEISS et al., 2000). Nesse caso, obtém-se o FEB a partir da relação entre a biomassa da parte aérea e a biomassa do fuste da árvore. Ainda, se existir a necessidade de realizar a estimativa incluindo a parte das raízes, recorre-se ao cálculo da razão de raízes (R). Essa razão é obtida através da relação entre a biomassa das raízes e a biomassa aérea.

Existem também algumas maneiras de utilizar o FEB e o R. Pode-se lançar mão de valores fixos, conhecidos como default, como apresentado nos trabalhos de Kauppi et al. (1992), Kauppi et al. (1995), Lowe et al. (2000), UN-ECE/FAO (2000), Food and Agriculture Organization (FAO) (2001) e Intergovernmental Panel on Climate Change (IPCC) (2006), apesar de se saber que esses valores variam dependendo das espécies, fases do crescimento e sítio (SATOO; MADGWICK, 1982), ou, então, desenvolver ou utilizar modelos que tenham sido desenvolvidos para a realização dessas estimativas, como o trabalho de Bartelink (1997), Ter-Mikaelian; Korzukhin (1997) e Lehtonen et al. (2004).

Este estudo teve como objetivo avaliar a correlação das principais variáveis dendrométricas com o FEB e o R, bem como desenvolver equações matemáticas que expressem a relação alométrica entre as variáveis de fácil e difícil obtenção. Ainda, foi alvo deste trabalho comparar as estimativas de biomassa em três situações possíveis: utilizando FEB e R default do IPCC (2006), utilizando FEB e R médios obtidos por este trabalho e utilizando FEB e R obtidos através de equações matemáticas desenvolvidas neste trabalho.

\section{MATERIAL E MÉTODOS}

\section{Dados coletados em campo}

Os dados utilizados neste estudo advêm de 70 árvores das espécies Pinus elliottii e Pinus taeda, plantadas no sul do estado do Paraná, com idades variando entre 2 e 24 anos, DAP variando entre $1,91 \mathrm{~cm}$ e 40,27 cm e alturas variando entre $2,35 \mathrm{~m}$ e $30,3 \mathrm{~m}$. Essas árvores foram objeto de medições dendrométricas e quantificação de biomassa pelo método direto, por meio de determinação de seu peso verde (fresco) em campo. 
No campo, foram escolhidas árvores representativas por classes de idade e de DAP, buscando-se sempre selecionar indivíduos médios das condições locais. Foram medidas as circunferências à altura do peito (CAP) de cada indivíduo, utilizando-se fita métrica, as quais foram posteriormente transformadas em DAP (diâmetro à altura do peito). As árvores foram derrubadas e tiveram suas alturas totais $(\mathrm{H})$ medidas com uso de trena. A idade de cada indivíduo foi determinada por contagem de anéis de crescimento e registros do ano de plantio.

Durante a pesagem em campo, cada árvore foi separada em compartimentos, quais sejam: fuste, galhos acima de $4 \mathrm{~cm}$ de diâmetro, galhos inferiores a $4 \mathrm{~cm}$ de diâmetro, folhagem e raízes. Os procedimentos de determinação de biomassa fresca seguiram as recomendações do IPCC (2006). De cada compartimento, obteve-se o peso verde, determinado com balança mecânica com capacidade de até 100 quilos e precisão de 100 gramas. De cada compartimento foram selecionadas porções amostrais representativas da biomassa, com o objetivo de realizar as determinações de biomassa seca e os respectivos teores de carbono, em laboratório.

As amostras foram levadas para laboratório e então pesadas frescas (peso verde), bem como fracionadas em tamanhos menores, para favorecer a secagem em estufa até peso constante a $103^{\circ} \mathrm{C}$. As porções amostrais secas foram pesadas em balança eletrônica de precisão de $1 \mathrm{~g}$ e os percentuais de biomassa seca de cada compartimento foram calculados com base na relação:

$$
\% \text { Matéria Seca }=\left(\frac{\mathrm{PS}}{\mathrm{PV}}\right) * 100
$$

Em que: $\mathrm{PS}=$ peso seco total do compartimento $(\mathrm{g})$;

$\mathrm{PV}=$ peso verde do compartimento $(\mathrm{g})$.

A fórmula (1) foi utilizada para determinar os pesos secos totais de cada compartimento, pesados frescos em campo.

\section{Cálculo do fator de expansão de biomassa e razão de raízes}

Para o cálculo do fator de expansão de biomassa (FEB) das árvores, foi utilizada a fórmula (2), indicada pelo IPCC (2006):

$$
\mathrm{FEB}=\frac{\mathrm{P}_{\text {copa }}+\mathrm{P}_{\text {fuste }}}{\mathrm{P}_{\text {fuste }}}=\frac{\mathrm{P}_{\text {aéreo }}}{\mathrm{P}_{\text {fuste }}}
$$

Em que: $\mathrm{FEB}$ = fator de expansão da biomassa (adimensional);

$\mathrm{P}_{\text {copa }}=$ peso seco da copa da árvore $(\mathrm{g})$;

$\mathrm{P}_{\text {fuste }}=$ peso seco do fuste da árvore $(\mathrm{g})$;

$\mathrm{P}_{\mathrm{a} e \text { reo }}=$ peso seco do fuste da árvore + peso da copa da árvore $(\mathrm{g})$.

A razão de raízes foi calculada segundo a fórmula (3), também indicada pelo IPCC (2006):

$$
\mathrm{R}=\frac{\mathrm{P}_{\text {raiz }}}{\mathrm{P}_{\text {aéreo }}}
$$

Em que: $\mathrm{R}$ = razão de raízes (adimensional);

$\mathrm{P}_{\text {raiz }}=$ peso seco da raiz da árvore ( $\mathrm{g}$ );

$\mathrm{P}_{\text {aéreo }}=$ peso seco da parte aérea da árvore $(\mathrm{g})$.

Análise estatística e ajuste de modelos matemáticos

Com o objetivo de construir modelos estimativos do FEB e R, foi construída uma matriz de correlação linear simples com as informações de DAP, H, idade, FEB e R obtidas nas 70 árvores amostradas.

Foram selecionados 12 modelos matemáticos encontrados na literatura florestal aplicados para estimativa de biomassa e volume do fuste, os quais foram testados para a estimativa de FEB e R (Tabela 1). 
Tabela 1. Modelos testados para estimar o fator de expansão de biomassa.

Table 1. Models tested to estimate the biomass expansion factor.

\begin{tabular}{|c|c|c|c|}
\hline \multicolumn{2}{|c|}{ Modelos ajustados para FEB } & \multicolumn{2}{|c|}{ Modelos ajustados para $\mathbf{R}$} \\
\hline & $\mathrm{FEB}=\beta_{0} D A P^{-\beta_{1}}$ & & $\mathrm{R}=\beta_{0} D A P^{-\beta_{1}}$ \\
\hline & $\mathrm{FEB}=\beta_{0} H^{-\beta_{1}}$ & 2 & $\mathrm{R}=\beta_{0} H^{-\beta_{1}}$ \\
\hline 3 & $\mathrm{FEB}=\beta_{0} I D A D E^{-\beta}$ & 3 & $\mathrm{R}=\beta_{0} I D A D E^{-\beta_{1}}$ \\
\hline 4 & $\mathrm{FEB}=\beta_{0}+\beta_{1} L n \mathrm{QP}_{-}^{-}$ & 4 & $\mathrm{R}=\beta_{0}+\beta_{1} L n$ \\
\hline 5 & $\mathrm{FEB}=\beta_{0}+\beta_{1} L n$ & 5 & $\mathrm{R}=\beta_{0}+\beta_{1} \operatorname{Ln}$ \\
\hline 6 & $\mathrm{FEB}=\beta_{0}+\beta_{1} L n$ DADE & 6 & $\mathrm{R}=\beta_{0}+\beta_{1} L n$ \\
\hline & $\mathrm{FEB}=\beta_{0} * \operatorname{EXP} \beta_{1}{ }^{* \mathrm{DAP}}$ & & $\mathrm{R}=\beta_{0} E X P\left(\beta_{1} D A P\right.$ \\
\hline & 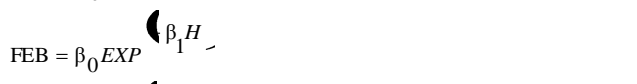 & 8 & 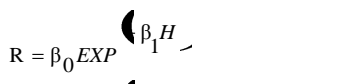 \\
\hline 9 & $\mathrm{FEB}=\beta_{0} E X P\left(\mathbf{\beta}_{1} I D A D E\right.$ & 9 & 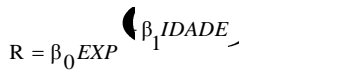 \\
\hline 10 & $\mathrm{FEB}=\beta_{0}+\beta_{1} D A P+\beta_{2} H+\beta_{3} I D A D E$ & 10 & $\mathrm{R}=\beta_{0}+\beta_{1} D A P+\beta_{2} H+\beta_{3} I D A D E$ \\
\hline 11 & $\mathrm{FEB}=\beta_{0}+\beta_{1} \ln (D A P H I D A D E)$ & 11 & $\mathrm{R}=\beta_{0}+\beta_{1} \ln (D A P H I D A D E)$ \\
\hline 12 & $F E B=\beta_{0}+\beta_{1} \ln D A P+\beta_{2} D A P+\beta_{3} \ln H+\beta_{4} \ln (D A P H$ IDADE $)$ & 12 & $\mathrm{R}=\beta_{0}+\beta_{1} \ln D A P+\beta_{2} \ln H$ \\
\hline
\end{tabular}

Em que: $\beta_{\mathrm{i}:}$ coeficientes dos modelos; FEB: Fator de Expansão de Biomassa; R: Razão de Raízes; ln: logaritmo neperiano; DAP: diâmetro a altura do peito (cm); H: altura total (m); EXP: exponencial.

Como critérios para seleção dos melhores ajustes, foram adotados o erro padrão da estimativa em porcentagem ( $\left.\mathrm{Syx}_{\%}\right)$, o coeficiente de determinação ajustado $\left(\mathrm{R}_{\mathrm{aj}}^{2}\right)$ e a análise gráfica dos resíduos.

\section{Comparação entre as estimativas da modelagem, valores médios e default do IPCC}

Tendo em vista que é usual utilizar valores constantes de FEB e R em projetos de MDL e em outros estudos que demandam quantificação do equivalente em $\mathrm{CO}_{2}$ contido nos povoamentos florestais, fez-se neste estudo uma análise comparativa dos resultados obtidos, aplicando-se os modelos selecionados de FEB e R. Para essa comparação, foi simulada a produção volumétrica de um hectare empregando-se o simulador SISPINUS (OLIVEIRA, 1995). O regime de manejo simulado considerou um plantio florestal com 1.667 árvores por hectare e índice de sítio igual a 23, com corte raso aos 18 anos, sem realização de desbastes. Para fins de comparação, foi utilizado o valor default de $\mathrm{FEB}=1,30$ e $\mathrm{R}=0,33$, valores sugeridos para Pinus pelo IPCC (2006).

A comparação realizada foi aplicando os cenários: $\mathbf{0}$ - FEB e R default do IPCC para estimativa de biomassa, carbono e $\mathrm{CO}_{2}$ eq; 2 - Valores médios de $\mathrm{FEB}$ e $\mathrm{R}$ observados na base de dados para estimativa de biomassa, carbono e $\mathrm{CO}_{2}$ eq; e 3 - Valores estimados por equação de FEB e $\mathrm{R}$ para estimativa de biomassa, carbono e $\mathrm{CO}_{2}$ eq. Os valores mais próximos dos verdadeiros são aqueles constatados pela base de dados utilizada, retratados pelos valores médios observados neste trabalho. Dessa forma, esses valores subsidiaram a referida comparação. Sendo assim, apesar de não ser o parâmetro da população, adotou-se essa estimativa média de FEB e R como valores referenciais para comparação.

Uma vez que o SISPINUS realiza a projeção por idade e diferentes informações dendrométricas, bem como que o modelo utilizado também tem como variável independente DAP e idade, essas variáveis foram levadas em consideração nesse cenário de comparação de desempenho dos métodos de estimativas.

A estimativa de biomassa e carbono a partir de FEB e R é obtida a partir da aplicação da seguinte expressão:

$$
\begin{gathered}
\text { Ptotal }=\text { Pfuste } * \mathrm{FEB} *(1+\mathrm{R}) \quad \text { ou } \\
\text { Carbono }=\text { Pfuste } * \mathrm{FEB} *(1+\mathrm{R}) * \mathrm{FC}
\end{gathered}
$$




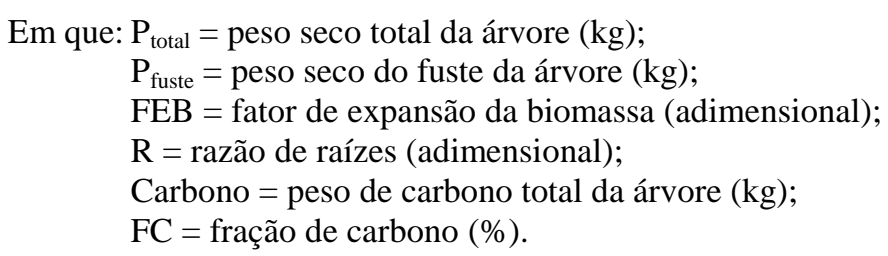

\section{RESULTADOS E DISCUSSÃO}

\section{Estatísticas descritivas das variáveis analisadas}

A tabela 2 apresentada abaixo sumariza as estatísticas descritivas das variáveis analisadas neste estudo. Nota-se que o valor médio de FEB foi de 1,47 para as condições deste estudo, variando de 1,09 a 3,74, ou seja, existe uma grande variabilidade nessa variável. No trabalho de Levy et al. (2010), para três espécies de coníferas, os valores de FEB variaram entre 1,04 e 2,32. Em um estudo de carbono desenvolvido para a Europa, Liski et al. (2002) utilizaram um valor FEB de 1,39 para floresta de coníferas do Reino Unido, com base em dados do Programa Internacional de Biologia e para a Alimentação e a Agricultura das Nações Unidas. Poucos outros valores estão disponíveis para comparação. Schroeder et al. (1997) estimou um valor de FEB de 1,25 para povoamentos de árvores adultas caducifólias nos Estados Unidos.

Tabela 2. Estatística descritiva dos dados observados de DAP, altura, idade, FEB e R.

Table 2. Descriptive statistics of the actual data DBH, tree height, age, BEF and R.

\begin{tabular}{lccccc}
\hline Estatísticas & DAP $(\mathbf{c m})$ & H $(\mathbf{m})$ & Idade $(\mathbf{a n o s})$ & FEB & R \\
\hline Média & 20,10 & 15,15 & 11,44 & 1,47 & 0,17 \\
Desvio padrão & 8,78 & 7,65 & 5,95 & 0,47 & 0,11 \\
Mínimo & 1,91 & 2,35 & 2 & 1,09 & 0,05 \\
Máximo & 40,27 & 30,30 & 24 & 3,74 & 0,63 \\
Coeficiente de variação & 43,78 & 50,99 & 51,75 & 33,33 & 50,00 \\
Contagem & 70 & 70 & 70 & 70 & 70 \\
\hline
\end{tabular}

$\mathrm{O}$ mesmo pode ser dito em relação a $\mathrm{R}$, que teve como média o valor 0,17 , o qual oscilou bastante, de um mínimo de 0,11 até 0,63. Isso que dizer que, em média, a copa representa 47\% da biomassa aérea e que a biomassa subterrânea corresponde, em média, a 17\% da biomassa total. Conforme será visto mais adiante, FEB e R têm correlação com as variáveis dendrométricas e com a idade, ou seja, à medida que as árvores mudam de dimensões com a idade, alteram-se as proporções de copa e de raízes nas duas espécies de Pinus consideradas. No estudo de Levy et al. (2010), o valor apontado como médio para o R foi de 0,36 , e Liski et al. (2002) utilizaram um valor menor de $\mathrm{R}(0,16)$ para floresta de coníferas do Reino Unido.

\section{Comportamento de FEB e R em função de DAP, H e idade}

Os resultados revelaram que o fator de expansão da biomassa e a razão de raízes variam de maneira considerável em função da dimensão da árvore e idade avaliada, haja vista as tendências apresentadas na figura 1 e a análise de correlação apresentada na tabela 3 .

A análise das relações entre o fator de expansão de biomassa e as variáveis DAP, H e idade revelou uma tendência bem definida de comportamento exponencial negativa, mostrando que, com o passar do tempo e o aumento das dimensões da árvore, os indivíduos apresentam uma redução acentuada na proporção da parte aérea e na de raízes. Esse comportamento se verifica em função de dois fenômenos: o amadurecimento fisiológico das árvores, que inicialmente requerem mais massa fotossintética e de raízes para promover o arranque inicial do crescimento; e a concorrência, que promove o sombreamento da copa e a desrama natural e a limitação da expansão das raízes, devido à ocupação do espaço vital por seus vizinhos competidores. 

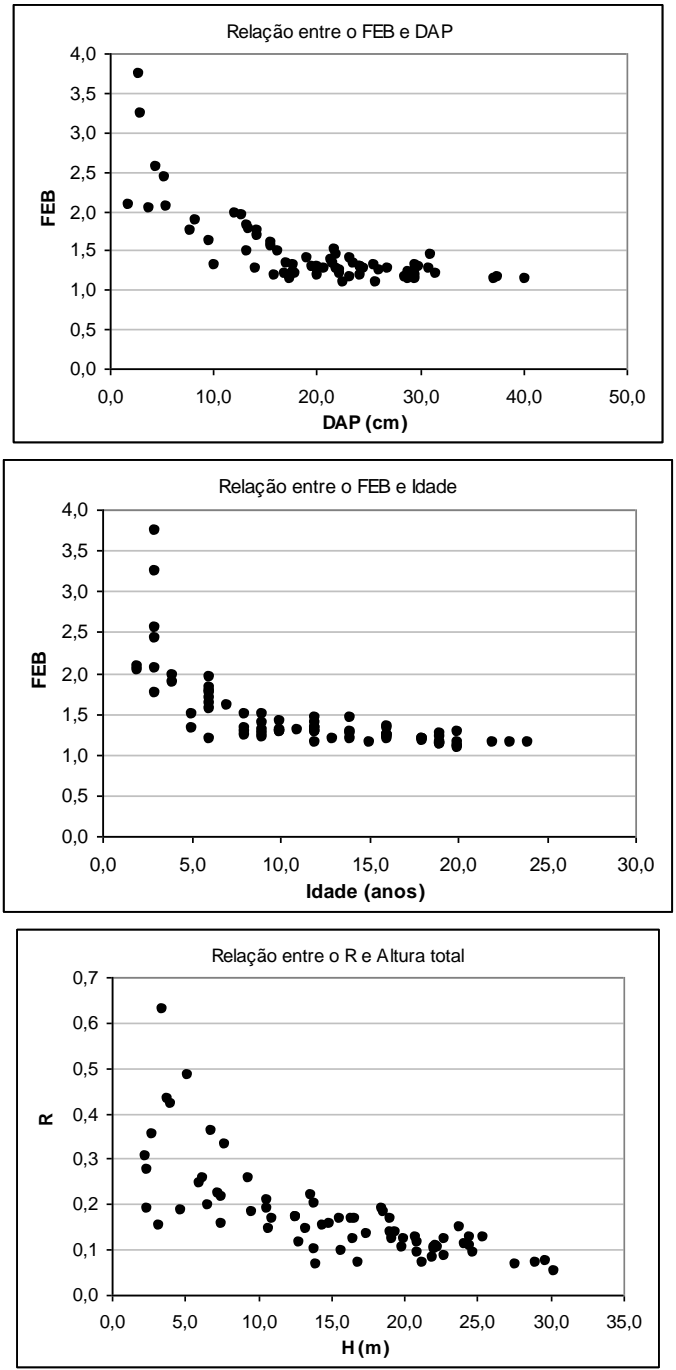
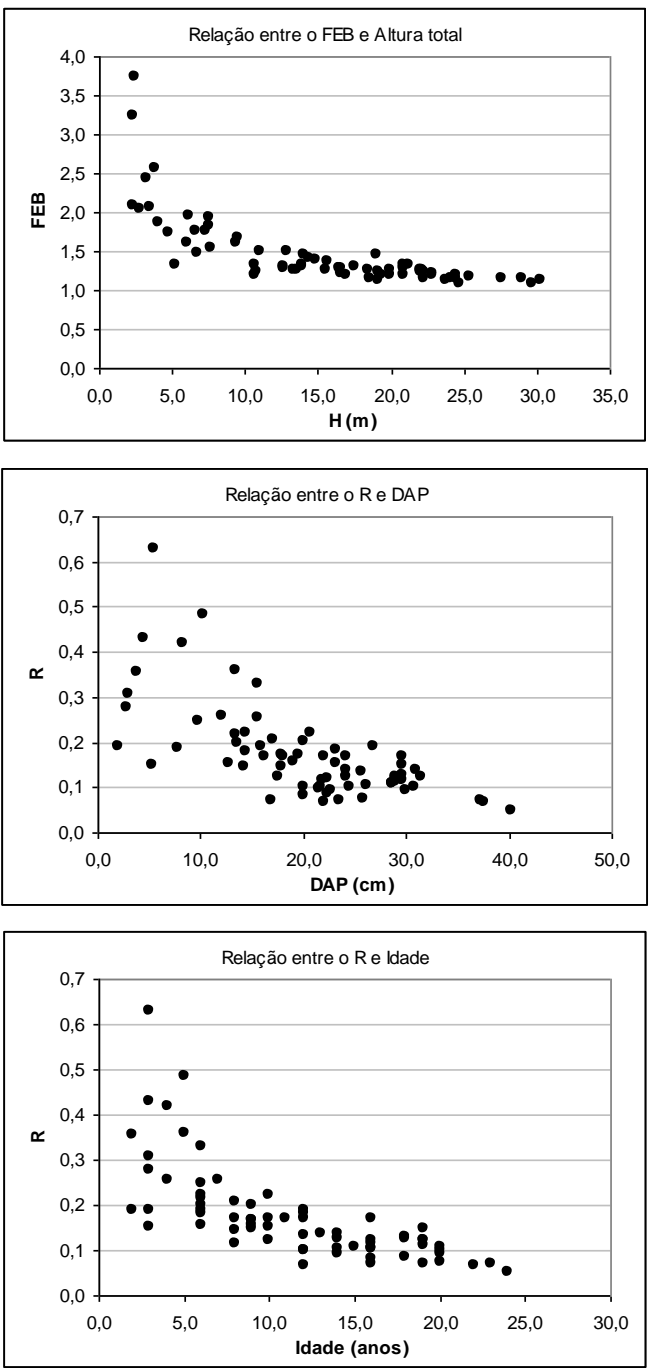

Figura 1. Relações entre as variáveis FEB, R, DAP, H e idade.

Figure 1. Relations between the variables $\mathrm{BEF}, \mathrm{R}, \mathrm{DBH}, \mathrm{H}$ and age.

Tabela 3. Matriz de correlação entre as variáveis FEB, R, DAP, H e idade.

Table 3. Correlation matrix between variables BEF, $\mathrm{R}, \mathrm{DBH}, \mathrm{H}$ and age.

\begin{tabular}{lccccc}
\hline Variáveis & $\begin{array}{c}\text { DAP } \\
(\mathbf{c m})\end{array}$ & $\begin{array}{c}\text { HT } \\
(\mathbf{m})\end{array}$ & $\begin{array}{c}\text { Idade } \\
(\mathbf{a n o s})\end{array}$ & FEB & $\mathbf{R}$ \\
\hline DAP $(\mathrm{cm})$ & 1 & - & - & - & - \\
HT $(\mathrm{m})$ & 0,922 & 1 & - & - & - \\
Idade (anos) & 0,869 & 0,960 & 1 & - & - \\
FEB & $-0,731$ & $-0,724$ & $-0,671$ & 1 & - \\
R & $-0,679$ & $-0,728$ & $-0,707$ & 0,528 & 1 \\
\hline
\end{tabular}

A tendência apresentada na figura 1 revela a redução em FEB e R com a idade e o tamanho das árvores (DAP e H), porém isso ocorre até certo ponto, pois, após um determinado ponto, verifica-se a sua estabilização, ou seja, existe uma tendência assintótica. Vê-se que a nuvem de pontos de FEB é menos dispersa que a de $\mathrm{R}$, reflexo da maior correlação da primeira variável com o diâmetro, a altura e a idade, como será visto com detalhes adiante. Essa tendência de diminuição da proporção de biomassa de raízes, 
bem como de ramos e folhas, ao longo do gradiente de idade também foi apontada em trabalhos desenvolvidos com essas variáveis, como Kauppi et al. (1995), Lehtonen et al. (2004), Brown (2002) e Fukuda et al. (2003).

Outra tendência observada neste trabalho é que em povoamentos mais jovens existe uma maior variação nos valores de FEB e R. Essa constatação também foi reportada no trabalho de Lehtonen et al. (2004). Dessa forma, para aplicação em projetos de MDL, deve-se procurar utilizar valores mais conservadores para povoamentos de menores idades. Isso é corroborado pela afirmação de Soares e Tomé (2004), que afirmam que, se não for possível a utilização de equações alométricas, pelo menos deve-se utilizar o uso do FEB, variando conforme classes de idade.

\section{Correlação entre FEB, R, DAP, H e idade}

A tabela 3 apresenta os resultados da matriz de correlação para as variáveis analisadas neste estudo. O coeficiente de correlação pode variar de $-1 \mathrm{a}+1$, e quanto maior o seu valor em módulo, maior o grau de associação entre duas variáveis. Quando o coeficiente for 0 não existe correlação entre as variáveis. Os dados da matriz de correlação indicam que o diâmetro é a variável que mais se associa ao FEB, seguido da altura.

A idade tem também correlação significativa, mas seu valor é inferior. Esses resultados demonstram que essas três variáveis explicam consideravelmente as variações em FEB. É, todavia, pertinente salientar que, por existir associação também entre DAP, $\mathrm{H}$ e idade, está embutida autocorrelação quando se consideram essas variáveis em conjunto na modelagem. Já para $R$, a variável mais correlata foi $\mathrm{H}$, ficando em segundo plano a idade e, em terceiro, o DAP. Nos estudos de Levy et al. (2010), Brown; Schroeder (1999) e Lehtonen et al. (2004), a altura total da árvore foi a variável mais útil para explicar as estimativas de FEB. Observa-se ainda uma tênue correlação entre FEB e R, o que pode ser explicado pela necessidade de a árvore requerer alometricamente maior biomassa de raízes para suportar proporcionalmente uma maior proporção de copa. Assim, é de se esperar que o FEB diminua com o aumento do tamanho da árvore, tendo em vista que a área foliar não aumenta na mesma proporção do porte da árvore. Tal comportamento é relatado no trabalho de Magnani et al. (2000).

\section{Modelos para estimar FEB e R em função de DAP, $H$ e idade}

Na tabela 4 estão demonstradas as equações ajustadas para estimar FEB e R, seus respectivos coeficientes e estatísticas de desempenho de ajuste.

De forma geral, as equações para estimar FEB resultaram em melhores ajustes que para a estimativa de R. Isso ocorreu devido à maior variabilidade da primeira em relação à segunda, expressa pelo coeficiente de variação, maior dispersão (como visto na Figura 1) e menor correlação com as variáveis dendrométricas e com a idade. Os coeficientes de determinação para FEB nas 12 equações variaram de 0,44 a 0,78 , enquanto que para $R$ oscilaram de 0,35 a 0,59 . No que diz respeito a $S_{y x} \%$, os valores para as equações de FEB estiveram entre 15,60 e 24,20\%, enquanto que para $\mathrm{R}$ esses valores ficaram compreendidos entre 39,72 e $48,84 \%$. Com base nos valores de $\mathrm{R}^{2}$ e $\mathrm{S}_{\mathrm{yx}} \%$, o modelo 12 foi o que teve o melhor desempenho para estimar FEB, porém os modelos $1,2,3$ e 11 tiveram comportamentos estatísticos próximos ao do modelo 12. De uma forma geral, o modelo 12 foi o que se mostrou melhor para FEB e para R, considerando esses dois índices de ajustamento.

Em função da grande variabilidade existente para o ajuste dos modelos, os erros encontram-se com erros representativos.

No que concerne à análise de resíduos dos dois conjuntos de equações, percebe-se, pelas figuras 2 e 3, que há uma maior dispersão de resíduos com as equações para estimativa de $\mathrm{R}$ do que com as equações para estimar FEB. Entre os modelos para FEB não houve diferenças expressivas em termos de distribuição de resíduos dos modelos, como visto na figura 2 . O mesmo pode ser dito em relação às equações para estimar $\mathrm{R}$, que não apresentaram tendência a superestimar ou a subestimar, tendo distribuição regular ao longo da abscissa do gráfico de resíduos. Entretanto, é importante destacar que a dispersão de resíduos nas equações para estimar $\mathrm{R}$ é muito superior àquelas para estimar FEB, como pode ser visto nas figuras 2 e 3 . Assim, os dois modelos 12, tanto para FEB como para R, foram considerados os melhores. 
Tabela 4. Grandezas estatísticas de ajuste dos modelos para estimar FEB e R.

Table 4. Statistical values of the fitted models for BEF and R.

\begin{tabular}{|c|c|c|c|c|c|c|c|c|}
\hline Modelo & $\boldsymbol{\beta}_{0}$ & $\beta_{1}$ & $\beta_{2}$ & $\boldsymbol{\beta}_{3}$ & $\boldsymbol{\beta}_{4}$ & $\mathbf{R}_{\text {aj }}^{2}$ & $\mathbf{S}_{\mathrm{yx}}$ & $\mathrm{S}_{\mathrm{yx}} \%$ \\
\hline \multicolumn{9}{|c|}{ Modelos ajustados para FEB } \\
\hline 1 & 3,9065 & $-0,3522$ & - & - & - & 0,72 & 0,25 & $16,92 \%$ \\
\hline 2 & 3,6190 & $-0,3697$ & - & - & - & 0,75 & 0,23 & $15,98 \%$ \\
\hline 3 & 3,2871 & $-0,3684$ & - & - & - & 0,75 & 0,23 & $15,96 \%$ \\
\hline 4 & 2,2597 & $-0,0392$ & - & - & - & 0,53 & 0,32 & $22,10 \%$ \\
\hline 5 & 2,1462 & $-0,0446$ & - & - & - & 0,52 & 0,33 & $22,45 \%$ \\
\hline 6 & 2,0792 & $-0,0532$ & - & - & - & 0,44 & 0,35 & $24,20 \%$ \\
\hline 7 & 2,5896 & 0,0301 & - & - & - & 0,62 & 0,29 & $20,02 \%$ \\
\hline 8 & 2,3734 & 0,0341 & - & - & - & 0,58 & 0,30 & $20,99 \%$ \\
\hline 9 & 2,3013 & 0,0421 & - & - & - & 0,50 & 0,33 & $22,94 \%$ \\
\hline 10 & 2,2313 & $-0,0215$ & $-0,0347$ & 0,0172 & - & 0,53 & 0,32 & $22,21 \%$ \\
\hline 11 & 3,0377 & $-0,2048$ & - & - & - & 0,70 & 0,26 & $17,89 \%$ \\
\hline 12 & 3,5663 & $-0,9055$ & 0,0296 & $-1,2032$ & 0,3844 & 0,78 & 0,22 & $15,60 \%$ \\
\hline \multicolumn{9}{|c|}{ Modelos ajustados para $\mathrm{R}$} \\
\hline 1 & 0,5389 & 0,4037 & - & - & - & 0,35 & 0,09 & $48,84 \%$ \\
\hline 2 & 0,5887 & 0,5006 & - & - & - & 0,51 & 0,07 & $42,42 \%$ \\
\hline 3 & 0,5830 & 0,5576 & - & - & - & 0,51 & 0,07 & $42,15 \%$ \\
\hline 4 & 0,4836 & $-0,1082$ & - & - & - & 0,43 & 0,08 & $45,53 \%$ \\
\hline 5 & 0,4662 & 0,1150 & - & - & - & 0,56 & 0,07 & $39,89 \%$ \\
\hline 6 & 0,4502 & 0,1215 & - & - & - & 0,55 & 0,07 & $40,47 \%$ \\
\hline 7 & 0,4017 & 0,0452 & - & - & - & 0,49 & 0,08 & $43,36 \%$ \\
\hline 8 & 0,3943 & 0,0609 & - & - & - & 0,57 & 0,07 & $39,72 \%$ \\
\hline 9 & 0,4003 & 0,0828 & - & - & - & 0,55 & 0,07 & $40,51 \%$ \\
\hline 10 & 0,3302 & 0,0008 & 0,0077 & 0,0020 & - & 0,53 & 0,07 & $42,14 \%$ \\
\hline 11 & 0,4800 & $-0,7298$ & - & - & - & 0,53 & 0,07 & $41,45 \%$ \\
\hline 12 & 0,4105 & 0,0901 & $-0,1945$ & - & - & 0,59 & 0,07 & $39,61 \%$ \\
\hline
\end{tabular}

As equações ajustadas neste estudo são aplicáveis para uma região em que a distribuição de diâmetro e características alométricas das árvores sejam similares às características das árvores deste estudo. Dessa maneira, caso existam divergências em tratamentos silviculturais que possam acarretar mudanças alométricas das árvores, pode existir variação nos valores apontados para FEB e R.

Outra possibilidade que não foi explorada neste trabalho e que poderá ser testada em futuros trabalhos é a adoção de classes etárias para a avaliação do desempenho para os ajustes testados.

Comparação entre as estimativas da modelagem, FEB e R médios e valores default do IPCC

Para efeito de demonstrar como é importante ter estimativas fidedignas de FEB e R, foi realizada uma comparação entre as estimativas de biomassa e carbono fixado, bem como $\mathrm{CO}_{2}$ equivalente, em um povoamento florestal com pínus de 1 hectare, utilizando simulação pelo programa SISPINUS (OLIVEIRA, 1995).

Os resultados da análise comparativa entre as estimativas de estoque em biomassa total (aérea e subterrânea), carbono fixado e equivalente em $\mathrm{CO}_{2}$ estão na tabela 5. Com base nas simulações de volume e biomassa do fuste, obtida pela multiplicação por $0,3817 \mathrm{~g} / \mathrm{cm}^{3}$, correspondente à densidade básica da madeira de Pinus aos 18 anos (SETTE JR. et al., 2006), e também pelos valores default do IPCC (2006) ( $\mathrm{FEB}=1,30$ e $\mathrm{R}=0,33)$, chegou-se a 337,87 t/ha, que corresponde a 138,36 t C/ha, com base em um teor médio de carbono de $41 \%$ (dados não publicados dos autores), ou a 507,33 t $\mathrm{CO}_{2} \mathrm{eq} / \mathrm{ha}$. Se, ao invés de utilizar os valores default do IPCC, fossem usados os valores médios de FEB e R deste estudo, as estimativas seriam de 351,33 t de biomassa seca/ha, 144,05 t C/ha e 528,17 t $\mathrm{CO}_{2}$ eq/ha. Considerando os valores estimados pelas equações de regressão para FEB e R ajustadas neste estudo, obter-se-iam 251,32 t de biomassa seca/ha, 103,04 t C/ha e 377,82 t $\mathrm{CO}_{2}$ eq/ha, respectivamente.

Os resultados acima demonstram que os projetos de MDL que usam FEB e R default do IPCC ou até mesmo valores médios calculados especificamente com dados coletados em campo podem conduzir a 
estimativas errôneas, que tendem a superestimar a quantidade de $\mathrm{CO}_{2}$ equivalente em povoamentos florestais de Pinus. Pelas tendências já apresentadas anteriormente, percebe-se que os valores de FEB e R médios representam melhor os indivíduos mais velhos e de maior porte do que os valores do IPCC.
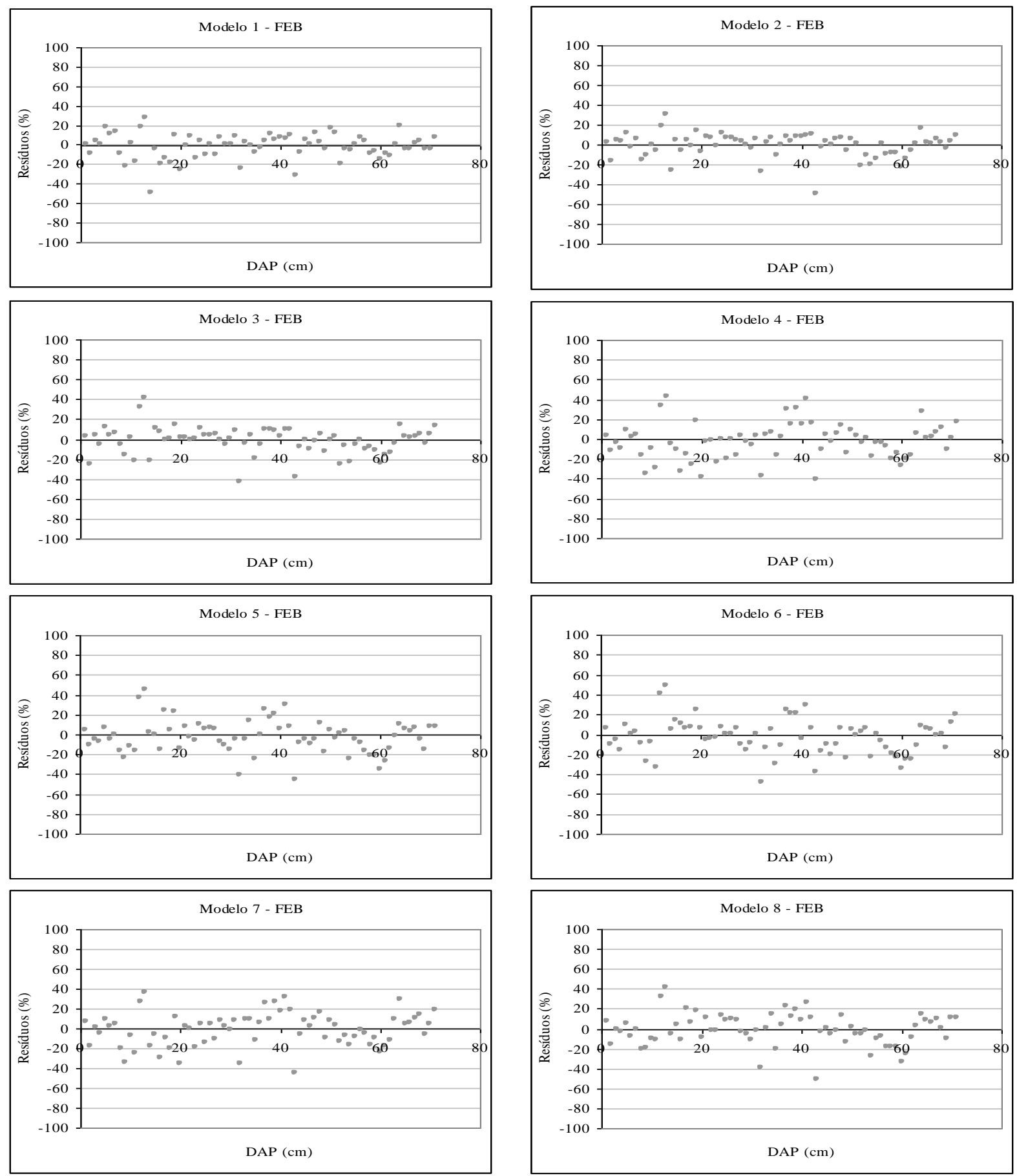

FLORESTA, Curitiba, PR, v. 42, n. 4, p. 755 - 768, out./dez. 2012. Corte, A. P. D.; Silva, F. da; Sanquetta, C. R. 

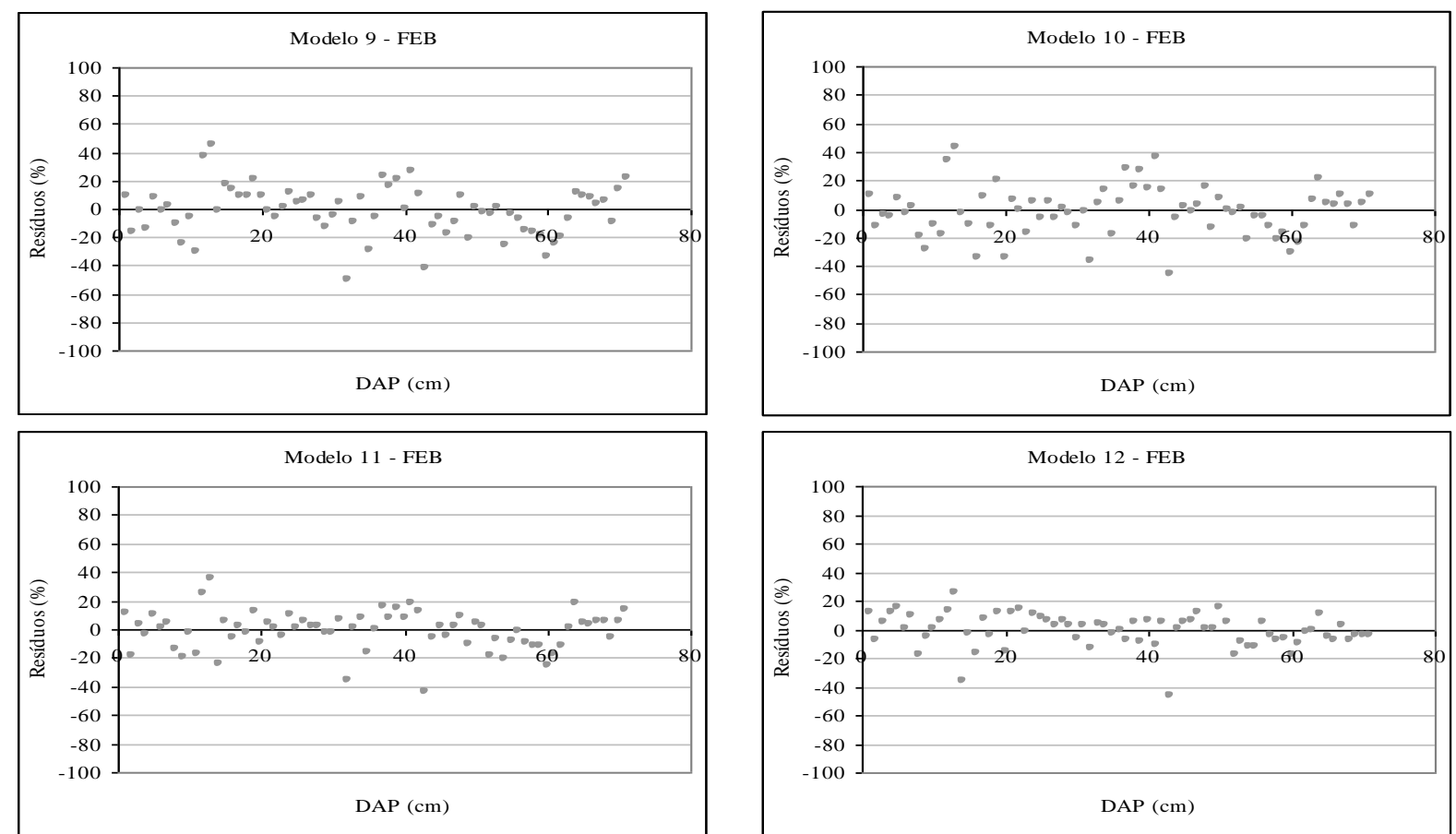

Figura 2. Distribuição gráfica de resíduos das equações ajustados para estimar FEB.

Figure 2. Graphical distribution of the residuals of the fitted models to estimate BEF.
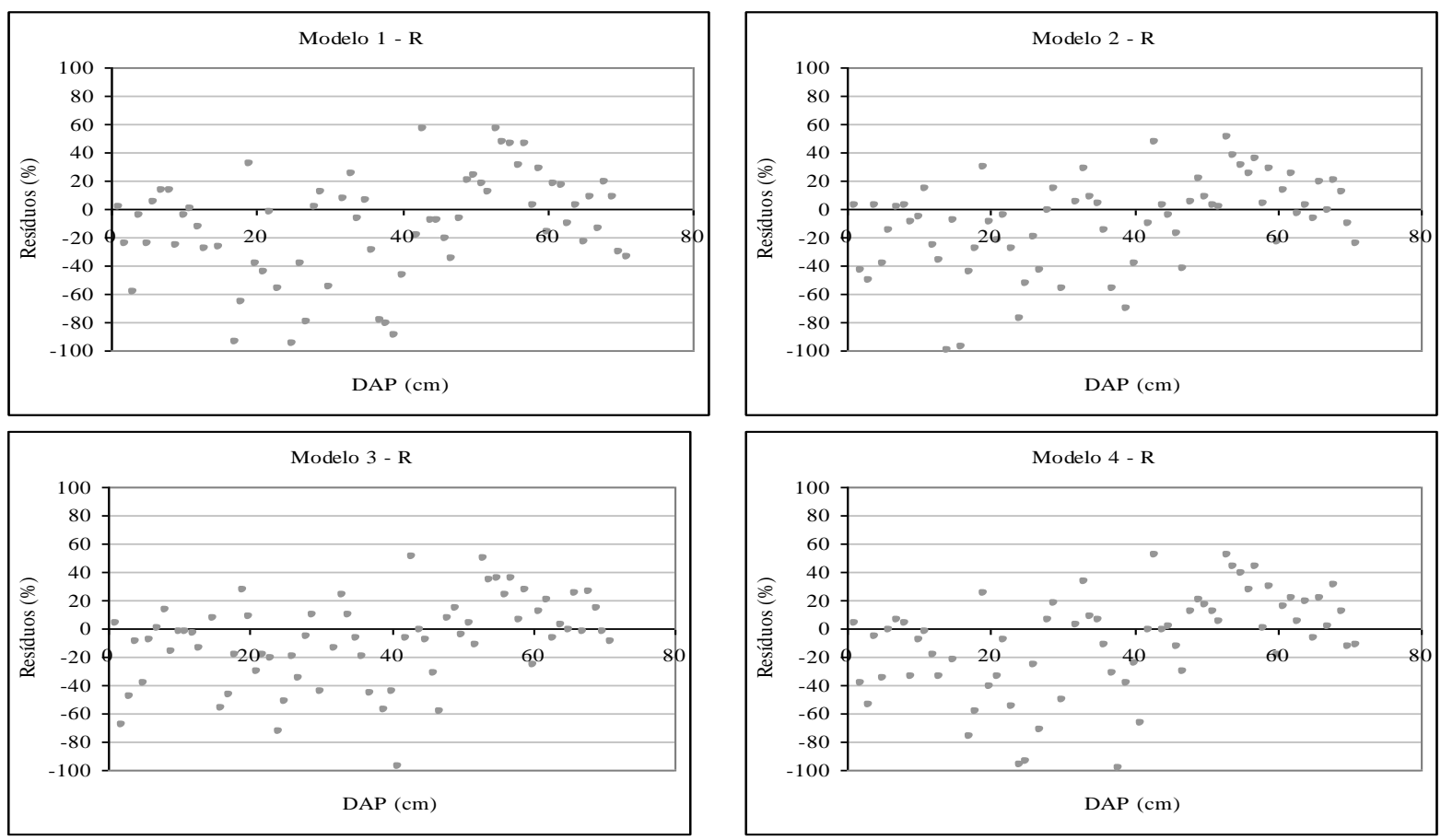

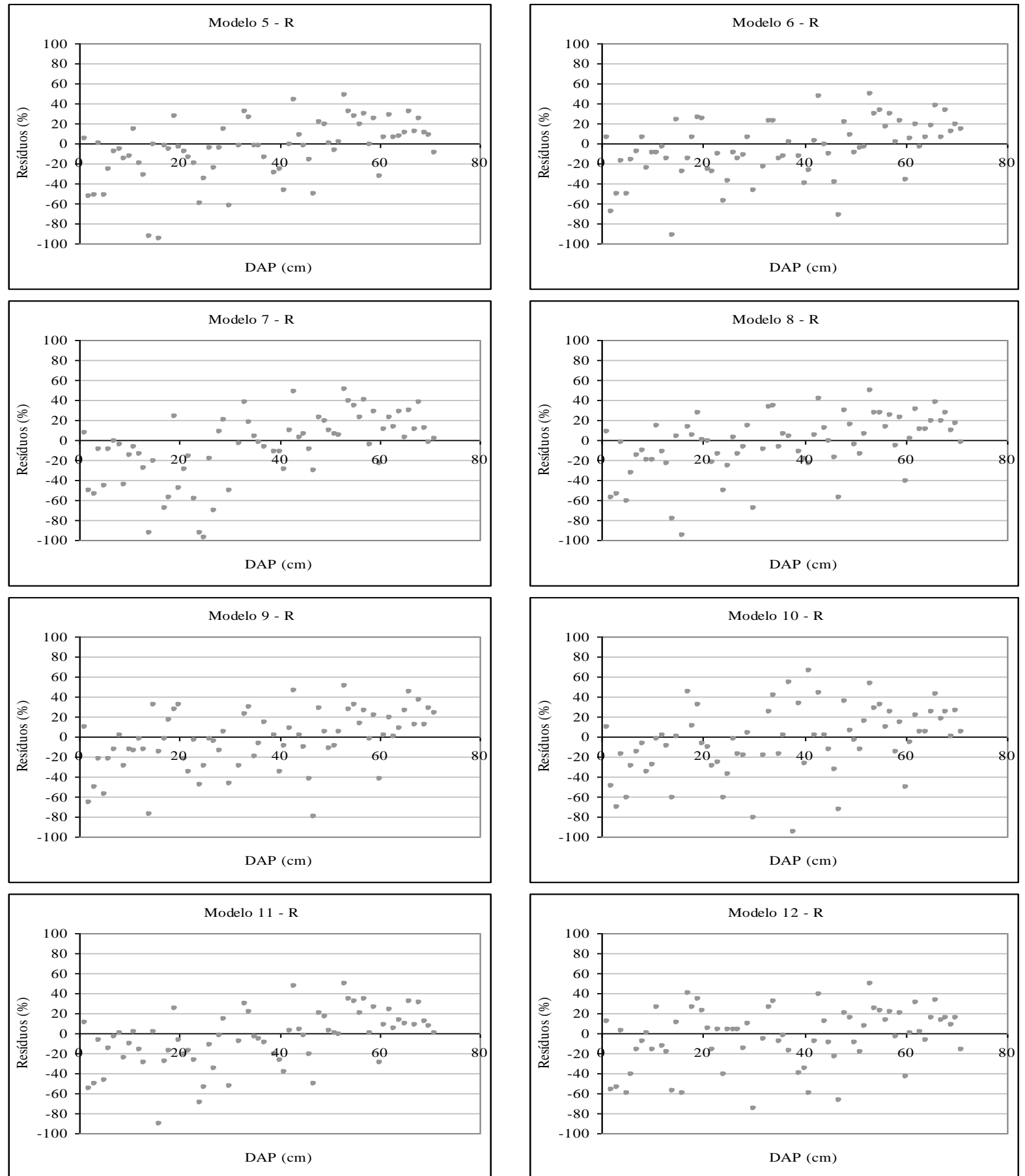

Figura 3. Distribuição gráfica de resíduos das equações ajustadas para estimar R.

Figure 3. Graphical distribution of the residuals of the fitted models to estimate R.

Essas superestimativas foram calculadas neste estudo em $28,46 \%$ quando se usam os valores default do IPCC e em 25,52\% quando se empregam os valores médios de FEB e R. Se essas comparações forem feitas com base na estimativa obtida com as equações, podem chegar a 34,28\% e 39,79\%, respectivamente. Se for considerado o valor referencial de US\$ 10.00/t $\mathrm{CO}_{2}$ eq, um câmbio de $\mathrm{R} \$ 1,80 / \mathrm{US} \$$ e uma área de 1.000 ha de um projeto hipotético de pequena escala no MDL, as 
superestimativas de receita com o projeto seriam da ordem de $\mathrm{R} \$ 2,3$ a 2,7 milhões. Além da falta de consistência na estimativa de receita com o projeto, o ganho ambiental em termos de captura de carbono também poderia estar sendo superdimensionado. Isso demonstra que pode estar havendo problemas metodológicos sérios nos projetos $\mathrm{A} / \mathrm{R}$ (Afforestation/Reforestation) de MDL e que é necessária a modelagem das variáveis FEB e R, considerando explicitamente sua correlação com o DAP, a altura e a idade das árvores do povoamento.

Tabela 5. Produção simulada aos 18 anos da biomassa seca total, do carbono fixado e do equivalente em $\mathrm{CO}_{2}$ em 1 ha de plantação de Pinus sp. com valores fixos de FEB e R e seus correspondentes, empregando os melhores modelos de regressão do presente estudo.

Table 5. Simulated yield at 18 years of total dry biomass, carbon stock and $\mathrm{CO}_{2}$ equivalent in a onehectare stand of Pinus sp. using constant values for FEB and R and the respective estimated by regression modeling.

\begin{tabular}{|c|c|c|c|}
\hline Variável & $\begin{array}{l}\text { FEB e R default } \\
\text { IPCC O }\end{array}$ & $\begin{array}{c}\text { FEB e R médios } \\
2\end{array}$ & $\begin{array}{c}\text { FEB e R equações } \\
3\end{array}$ \\
\hline Biomassa total (t/ha) & 337,47 & 351,33 & 251,32 \\
\hline Carbono estocado (t/ha) & 138,36 & 144,05 & 103,04 \\
\hline $\mathrm{CO}_{2}$ equivalente $(\mathrm{t} / \mathrm{ha})$ & 507,33 & 528,17 & 377,82 \\
\hline D - 2 & & & \\
\hline Diferença $(\%) 2$ - 3 & & \multirow{2}{*}{\multicolumn{2}{|c|}{25,53}} \\
\hline (1 - 3 & & & \\
\hline
\end{tabular}

\section{CONCLUSÕES}

Com base nos resultados obtidos neste trabalho, pode-se concluir o que segue:

- O fator de expansão da biomassa (FEB) e a razão de raízes (R) são correlacionados às variáveis diâmetro a 1,30 m, altura total e idade das árvores e variam de forma significativa conforme valores destes, tendendo para uma curva exponencial negativa até certo ponto, em que passa a ter uma tendência assintótica.

- Os modelos matemáticos podem ser utilizados para explicar a relação entre o FEB e R com as variáveis dendrométricas, sendo o modelo 12 o que apresentou os melhores indicadores de ajuste neste estudo.

- $\quad$ Em projetos de MDL, FEB e R são grandezas fundamentais para se obterem estimativas confiáveis dos créditos de carbono gerados. Observando os resultados deste estudo, vê-se que, ao utilizar os valores default do IPCC (2006) em comparação com os obtidos com as equações ajustadas, ocorrem divergências de resultados da ordem de $28,46 \%$ nas estimativas de $\mathrm{CO}_{2}$ eq fixadas pelo povoamento florestal. Utilizando os dados médios de FEB e R deste trabalho aplicados aos dados simulados, percebe-se que, ainda assim, as divergências podem chegar à casa de 25,53\%. Dessa forma, concluise que não devem ser utilizados valores default para tais estimativas, tendo em vista as sérias implicações que elas poderão trazer quando do desenvolvimento de um projeto de MDL.

\section{REFERÊNCIAS}

BARTELINK, H. H. Allometric relationships for biomass and leaf area of beech (Fagus sylvatica L.). Ann. For. Sci., v. 54, p. 39 - 50. 1997.

BROWN, S. Measuring carbon in forests: current status and future challenges. Environ. Pollut., v. 116, p. 363 - 372. 2002.

BROWN, S. L.; SCHROEDER, P. E. Spatial patterns of aboveground production and mortality of woody biomass for Eastern US forests. Ecol. Applicat., v. 9, p. 968 - 980. 1999.

FERNANDES, L. A. V. Customização da ferramenta "TARAM" para projetos de MDL florestal com Pinus taeda no extremo sul do Paraná. Pós-Graduação em Engenharia Florestal (Dissertação - Mestrado em Ciências Florestais) - Setor de Ciências Agrárias, Universidade Federal do Paraná, Curitiba, 2010. 152 p. 
FOOD AND AGRICULTURE ORGANIZATION (FAO). Global Forest Resource Assessment 2000. FAO Forestry Paper 140. FAO, Rome. 2001.

FUKUDA, M.; IEHARA, T.; MATSUMOTO, M. Carbon stock estimates for sugi and hinoki forests in Japan. For Ecol. Manage. v. 184, p. 1 - 16. 2003.

INTERGOVERNMENTAL PANEL ON CLIMATE CHANGE (IPCC). Guidelines for National Greenhouse Gas Inventories. Em CD ou no site: www.ipcc.ch. Guia de Boas Práticas. 2006.

JOHNSON, W. C.; SHARPE, D. M. The ratio of total to merchantable forest biomass and its application to the global carbon budget. Can. J. For. Res., v. 13, p. 372 - 383. 1983.

KARJALAINEN, T.; KELLOMÄKI, S. Greenhouse gas inventory for land use changes and forestry in Finland based on international guidelines. Mitigation Adapt. Strategies Global Climate, n. 1, p. 51 - 71. 1996.

KAUPPI, P. E.; MIELIKÄINEN, K.; KUUSELA, K. Biomass and carbon budget of European forests, 1971-1990. Science, v. 256, p. 70 - 74. 1992.

KAUPPI, P. E.; KAUPPI, E.; TOMPPO, E.; FERM, A. C and N storage in living trees within Finland since 1950s. Plant Soil, 168/169. p. 633 - 638. 1995.

LEHTONEN, A.; MÄKIPÄÄ, R.; HEIKKINEN, J.; SIEVÄNEN, R.; LISKI, J. Biomass expansion factors (BEFs) for Scots pine, Norway spruce and birch according to stand age for boreal forests. Forest Ecology and Management, v. 188, n. 1(3), p. 211 - 224. 2004.

LEVY, P. E.; HALE, S. E.; NICOLI, B. C. Biomass expansion factors and root: shoot ratios for coniferous tree species in Great Britain. Forestry, v. 77, n. 5, p. 421 - 430. 2010.

LISKI, J.; PERRUCHOUD, D.; KARJALAINEN, T. Increasing carbon stocks in the forest soils of Western Europe. For. Ecol. Manage., v. 169, p. 163 - 179. 2002.

LÖWE, H.; SEUFERT, G.; RAES, F. Comparison of methods used within member states for estimating $\mathrm{CO}_{2}$ emissions and sinks according to UNFCCC and EU monitoring mechanism: forest and other wooded land. Biotechnol. Agron. Soc. Environ., v. 4, p. 315 - 319. 2000.

MAGNANI, F.; MENCUCCINI, M.; GRACE, J. Age-related decline in stand productivity: the role of structural acclimation under hydraulic constraints. Plant Cell Environ., v. 23, p. 251 - 263. 2000.

OLIVEIRA, E. B. Um sistema computadorizado de prognose do crescimento e produção de Pinus taeda L., com critérios quantitativos para avaliação técnica e econômica de regimes de manejo. (Tese - Doutorado em Ciências Florestais) - Setor de Ciências Agrárias, Universidade Federal do Paraná, Curitiba, 1995. $134 \mathrm{p}$.

SANQUETTA, C. R.; CORTE, A. P. D.; BALBINOT, R.; ZILLIOTTO, M. A. B. Proposta metodológica para quantificação e monitoramento do carbono estocado em florestas plantadas. In: Carlos Roberto Sanquetta; Marco Aurélio Zilliotto (orgs.). Mercado de carbono: mercado e ciência. Curitiba: UFPR, v. 1 , p. 120 - 150. 2004.

SATOO, T.; MADGWICK, H. A. I. Forest biomass. Forestry Sciences. Martinus Nijhoff/Dr. W. Junk Publisher, The Hague. 152 p. 1982.

SCHROEDER, P.; BROWN, S.; MO, J.; BIRDSEY, R.; CIESZEWSKI, C. Biomass estimation for temperate broadleaf forests of the United States using inventory data. For. Sci., v. 43, p. 424 - 434. 1997.

SETTE JR., C. R.; NAKAJIMA, N. Y.; GEROMINI, M. P. Captura de carbono orgânico em povoamentos de Pinus taeda L. na região de Rio Negrinho, SC. Floresta, Curitiba, v. 36, n. 1, p. 33 - 44. 2006.

SOARES, P.; TOMÉ, M. Analysis of the effectiveness of biomass expansion factors to estimate stand biomass. In: HASENAUER, H.; MAKELA, A. (eds.). Modeling forest production. Proc. Conf. Vienna, 19-21 April (Department of Forest and Soil Sciences, BOKU University of Natural Resources and 
Applied Life Sciences, Vienna. p. 368 - 374. 2004.

TER-MIKAELIAN, M. T.; KORZUKHIN, M. D. Biomass equations for sixty-five North American tree species. For. Ecol. Manage., v. 97. p. 1 - 24. 1997.

UN-ECE/FAO, 2000. UN-ECE/FAO. Forest Resources of Europe, CIS, North America, Australia, Japan and New Zealand (industrialized temperate/boreal countries), UN-ECE/FAO Contribution to the Global Forest Resources Assessment 2000, Main Report United Nations, New York, Geneva. 2000.

WEISS, P.; SCHIELER, K.; SCHADAUER, K.; RADUNSKY, K.; ENGLISCH, M. Die Kohlenstoffbilanz des österreichischen Waldes und Betrachtungen zum Kyoto-Protokoll, Series Die Kohlenstoffbilanz des österreichischen Waldes und Betrachtungen zum Kyoto-Protokoll. Federal Environment Agency, Wien. 2000. 